\title{
PRODUÇÃO DE BIODIESEL VIA TRANSESTERIFICAÇÃO ETÍLICA COM ZEÓLITAS BÁSICAS
}

\author{
Grace F. Ghesti* \\ Engenharia de Energia, Faculdade UnB-Gama, Universidade de Brasília, 72405-610 Brasília - DF, Brasil \\ Julio L. de Macedo, José A. Dias e Sílvia C. L. Dias \\ Instituto de Química, Universidade de Brasília, CP 4478, 70904-970 Brasília - DF, Brasil
}

Recebido em 25/2/11; aceito em 30/6/11; publicado na web em 5/8/11

\begin{abstract}
BIODIESEL PRODUCTION VIA ETHYLIC TRANSESTERIFICATION WITH BASIC ZEOLITES. Soybean oil transesterification with ethanol was carried out in a batch reactor using USY zeolites modified with barium and strontium (15 wt.\%) as catalysts. A series of three catalytic cycles were performed for each zeolite without any loss of activity. The biodiesel product was analyzed by HPLC and FT-Raman, and the catalysts by pyridine and $\mathrm{CO}_{2}$ adsorption. Ba/USY provided higher conversions (> 97\%) than $\mathrm{Sr} / \mathrm{USY}(<$ $75 \%)$. The increased catalytic activity of Ba/USY was attributed to two different effects: a larger number of basic sites; and a lower interaction between barium species and HUSY Brønsted sites.
\end{abstract}

Keywords: biodiesel; basic zeolites; transesterification.

\section{INTRODUÇÃO}

A Sociedade Americana de Testes e Materiais (ASTM) definiu biodiesel como um combustível constituído de ésteres de monoalquila de ácidos graxos de cadeias longas derivados de óleos vegetais ou gordura animal, que satisfaçam os requerimentos da norma ASTM D $6751 .^{1,2} \mathrm{O}$ biodiesel apresenta diversas vantagens quando comparado ao diesel derivado de petróleo: é derivado de fontes renováveis, biodegradável, não tóxico, apresenta baixa emissão de poluentes, alto ponto de fulgor, excelente lubricidade e pode ser usado puro ou misturado com diesel derivado do petróleo. ${ }^{1,3,4}$

O uso de óleos vegetais como combustível é conhecido desde a Exposição de Paris, em 1900. ${ }^{4}$ No entanto, devido ao seu alto peso molecular e alta viscosidade cinética, seu uso direto em motores a diesel resultou em diversos problemas operacionais (e.g., pobre atomização, formação de depósitos de carbono devido à combustão incompleta, problemas de lubrificação, etc.). ${ }^{1,45}$ Para resolver estes problemas, quatro soluções possíveis foram investigadas na literatura: transesterificação (também chamada de alcoólise), pirólise, diluição com diesel derivado de petróleo e microemulsão. ${ }^{1,4-12}$

Dentre os métodos citados acima, a reação de transesterificação é o único processo que leva aos produtos definidos pela ASTM (i.e., ésteres de alquila) e é o método mais utilizado para produzir biodiesel. ${ }^{1-4} \mathrm{Na}$ transesterificação, as moléculas de triglicerídeos encontradas no óleo ou gordura animal ou vegetal (e.g., óleo de soja, ${ }^{12-14}$ amendoim, ${ }^{12}$ canola, ${ }^{1}$ etc.) reagem com um álcool (e.g., metanol, ${ }^{12}$ etanol, ${ }^{12-14}$ etc.) na presença de um catalisador (e.g., $\mathrm{NaOH},{ }^{12-14}$ $\mathrm{H}_{2} \mathrm{SO}_{4},{ }^{12}$ lipase, ${ }^{11}$ etc.) para formar ésteres e glicerol. ${ }^{1,3-5,10-12}$ Além das vantagens mencionadas anteriormente, o biodiesel produzido por transesterificação apresenta também propriedades similares ao diesel derivado de petróleo (e.g., número de cetano, viscosidade, massa molecular, densidade, etc. $)^{15-18}$ e não demanda nenhuma alteração nos motores a diesel. ${ }^{19}$

No Brasil, o etanol é um álcool mais barato que o metanol devido à sua alta produção a partir da biomassa da cana-de-açúcar. Neste sentido, o biodiesel produzido com etanol (ésteres de etila) pode ser visto como um combustível totalmente renovável. O uso de catalisadores heterogêneos para a produção de biodiesel por

*e-mail: grace@unb.br transesterificação pode eliminar alguns dos custos de processos adicionais associados a catalisadores homogêneos (e.g., corrosão, neutralização, decomposição do catalisador, etc.). Diversos estudos envolvendo o uso de catalisadores sólidos na reação de transesterificação são reportados na literatura: resina aniônica, ${ }^{20}$ óxidos (e.g., $\mathrm{SnO}, \mathrm{MgO}, \mathrm{ZnO},{ }^{21,22}$ etc.), óxidos suportados (e.g. $\mathrm{WO}_{3} / \mathrm{ZrO}_{2} / \mathrm{Al}_{2} \mathrm{O}_{3},{ }^{23} \mathrm{KNO}_{3} / \mathrm{Al}_{2} \mathrm{O}_{3},{ }^{24} \mathrm{NaOH} / \gamma-\mathrm{Al}_{2} \mathrm{O}_{3},{ }^{25}$ etc.), óxidos sulfatados $\left(\mathrm{SO}_{4}{ }^{2-} / \mathrm{ZrO}_{2}, \mathrm{SO}_{4}{ }^{2-} / \mathrm{SnO}_{2}\right),{ }^{26}$ materiais mesoporosos ${ }^{27} \mathrm{e}$ zeólitas ( $\mathrm{NaOx} / \mathrm{NaX}, \mathrm{ETS}-10,{ }^{21}$ etc. $)$, entre outros.

Dentre os catalisadores citados acima, as zeólitas se destacam devido à grande quantidade de aplicações que apresentam na indústria química, como catalisadores e adsorventes. As zeólitas apresentam propriedades singulares, tais como, alta área superficial, propriedades de adsorção, propriedades de troca iônica, centros ativos dentro do sistema de canais e cavidades, e diferentes tipos de seletividade..$^{28-34}$ A alta área superficial desses materiais e suas propriedades de troca iônica permitem que sua rede cristalina seja usada para gerar centros básicos ativos na estrutura zeolítica (e.g., troca iônica com sais de metais alcalinos, etc. $)^{35}$ ou como suporte para espécies básicas (e.g., hidróxidos, cátions ou óxidos básicos, etc.). ${ }^{36}$

Neste trabalho, teve-se como objetivo a preparação de zeólitas básicas através da modificação de zeólitas USY com bário e estrôncio e sua aplicação na reação de transesterificação de óleo de soja com etanol, para produção de ésteres de etila (biodiesel). Além disso, foram estudados os efeitos da geração de sítios básicos na superfície dos catalisadores, bem como suas respectivas atividades por três ciclos catalíticos. O biodiesel obtido nos ciclos foi analisado por HPLC, CG-FID e espectroscopia Raman. Já os catalisadores foram caracterizados por FTIR, análises térmicas e adsorção gasosa de piridina e $\mathrm{CO}_{2}$.

\section{PARTE EXPERIMENTAL}

\section{Materiais}

Piridina (Vetec) foi destilada sobre $\mathrm{CaH}_{2}$ (Merck) e depois armazenada sobre peneira molecular 3A (Aldrich). A zeólita USY (CBV 500) na forma amoniacal foi obtida da Zeolyst International, com as seguintes características: razão molar $\mathrm{SiO}_{2} / \mathrm{Al}_{2} \mathrm{O}_{3}=2,6$, área superficial $=750 \mathrm{~m}^{2} \mathrm{~g}^{-1}$ e $0,2 \%$ de $\mathrm{Na}_{2} \mathrm{O}$ em massa. Para os 
procedimentos de impregnação utilizou-se $\mathrm{Ba}\left(\mathrm{NO}_{3}\right)_{2}$ (Carlo Erba) e $\mathrm{SrCl}_{2} \cdot 6 \mathrm{H}_{2} \mathrm{O}$ (Vetec). Óleo de soja refinado (Soya ${ }^{\mathrm{TM}}$ ) foi usado como recebido. Etanol (Vetec, 99,8\%) foi tratado com peneira molecular $3 \mathrm{~A}$ (Aldrich) por $24 \mathrm{~h}$ antes dos experimentos.

\section{Preparação dos catalisadores}

As zeólitas Ba/USY e Sr/USY foram preparadas por impregnação em fase aquosa. Adicionou-se a zeólita $\mathrm{NH}_{4} \mathrm{USY}$ a uma solução de nitrato de bário ou cloreto de estrôncio contendo a quantidade necessária para preparar o catalisador com $15 \%$ de $\mathrm{BaO}$ ou $\mathrm{SrO}$ em massa. A suspensão foi, então, aquecida a $80^{\circ} \mathrm{C}$, mantida sob agitação até a evaporação total da água e, depois, o sólido resultante foi calcinado em um forno a $550{ }^{\circ} \mathrm{C}\left(10{ }^{\circ} \mathrm{C} \mathrm{min}-1\right)$ por $8 \mathrm{~h}$.

\section{Adsorção de piridina}

A adsorção de piridina em fase gasosa foi realizada simultaneamente para ambas as amostras, de acordo com procedimento reportado por nosso grupo. ${ }^{37}$ Os catalisadores foram desidratados em $\mathrm{N}_{2}$ anidro a $300{ }^{\circ} \mathrm{C}$ por $1 \mathrm{~h}$, resfriados a $100{ }^{\circ} \mathrm{C}$ e depois permitiu-se que piridina gasosa diluída em $\mathrm{N}_{2}$ passasse pelas amostras por $1 \mathrm{~h}$. Por fim, manteve-se a temperatura a $100{ }^{\circ} \mathrm{C}$ em fluxo de $\mathrm{N}_{2}$ por $1 \mathrm{~h}$ para remover a piridina fisicamente adsorvida. Após o experimento, as amostras foram analisadas por TG/DTG e FTIR. Os valores determinados desta forma foram idênticos aos obtidos para amostras ativadas em reator de vidro $\left(300{ }^{\circ} \mathrm{C} / 4 \mathrm{~h} /\right.$ vácuo $)$, manipuladas em câmara seca $\left(\mathrm{N}_{2}\right)$ e submetidas à adsorção em fase líquida de piridina diluída em cicloexano, evidenciando que a exposição das amostras ao ar antes das medidas de TG/DTG e FTIR não influenciou os resultados reportados.

\section{Adsorção de $\mathrm{CO}_{2}$}

A adsorção de $\mathrm{CO}_{2}$ foi realizada em um reator de vidro fabricado no laboratório. $\mathrm{O}$ catalisador foi adicionado ao reator, desidratado em vácuo a $300{ }^{\circ} \mathrm{C}$ por $1 \mathrm{~h}$, resfriado a $100{ }^{\circ} \mathrm{C}$ em vácuo e depois se permitiu que $\mathrm{CO}_{2}$ passasse pela amostra por $1 \mathrm{~h}$. Por fim, manteve-se a temperatura a $100{ }^{\circ} \mathrm{C}$ em vácuo por $1 \mathrm{~h}$ para remover $\mathrm{CO}_{2}$ fisicamente adsorvido. Após o fim do experimento, as amostras foram analisadas por TG/DTG.

\section{Difração de raios $\mathbf{X}$}

A difração de raios $\mathrm{X}$ (DRX) foi obtida a $1^{\circ} \mathrm{min}^{-1}$ em um difratômetro da Bruker, modelo D8 Focus, com radiação CuKo a $40 \mathrm{kV}$ e $30 \mathrm{~mA}$. O tamanho médio de cristalito $(D)$ foi determinado pela Equação de Sherrer, ${ }^{38}$ assumindo cristalitos esféricos:

$$
t=(4 / 3) k \lambda / \beta \cos \theta
$$

onde, $\theta$ é o ângulo de difração, $k$ é uma constante igual a 0,89 , $\lambda=0,15418 \mathrm{~nm}$ e $\beta$ é a largura a meia-altura (em radianos) do pico mais intenso.

\section{Medidas de FTIR}

Espectros de FTIR da região estrutural e após os experimentos de adsorção foram obtidos em um espectrômetro Bomen MB-100 usando-se pastilhas de $\mathrm{KBr}$. Os espectros foram adquiridos a $4 \mathrm{~cm}^{-1}$ de resolução após o acúmulo de 32 varreduras. As razões $\mathrm{Si} / \mathrm{Al}$ da rede para as zeólitas $\mathrm{Y}$ foram calculadas utilizando-se a equação reportada por Lutz e colaboradores (Equação 2): ${ }^{39}$

$$
x=3,857-0,00621 w_{D R}
$$

para $0,1<\mathrm{x}<0,3$, onde $w_{D R}$ é o modo de vibração dos anéis duplos na região entre 570 e $600 \mathrm{~cm}^{-1}$ e $x=[1+(\text { Si/Al })]^{-1}$.

\section{Análises térmicas}

As curvas de termogravimetria (TG) e termogravimetria derivada (DTG) foram obtidas em um 2960 Simultaneous DSC-TGA (TA Instruments) usando $\mathrm{N}_{2}(99,999 \%)$ como gás de purga $\left(100 \mathrm{~mL} \mathrm{~min}^{-1}\right)$. As análises foram realizadas da temperatura ambiente até $1000{ }^{\circ} \mathrm{C}$ a uma razão de $10{ }^{\circ} \mathrm{C} \mathrm{min}^{-1}$.

\section{Ensaio catalítico}

A atividade catalítica dos catalisadores foi testada na reação de transesterificação do óleo de soja com etanol. As zeólitas Ba/USY $(D=93 \mathrm{~nm})$ e Sr/USY $(D=95 \mathrm{~nm})$ foram ativadas nas mesmas condições utilizadas em sua caracterização antes de serem adicionadas ao meio reacional. As reações foram realizadas em um reator de aço inoxidável de alta pressão da Berghof (HR-200) com paredes de Teflon ${ }^{\circledR}$ acoplado a um regulador de temperatura e agitação. A razão molar utilizada para as reações foi 1:30:0,1 (óleo:álcool:catalisador), perfazendo um volume reacional de $30 \mathrm{~mL}$. O sistema foi mantido sob agitação de $1000 \mathrm{rpm}$, a $200{ }^{\circ} \mathrm{C}$ por 24 h e pressão autógena. Ao final desta etapa, o reator foi resfriado a temperatura ambiente, os catalisadores recuperados mediante filtração e o biodiesel obtido lavado três vezes com solução $5 \%$ de $\mathrm{NaCl}$ e seco com sulfato de magnésio anidro. Os catalisadores recuperados foram novamente calcinados a $550^{\circ} \mathrm{C}$ por $8 \mathrm{~h}$ para serem reutilizados nos ciclos seguintes. Três ciclos catalíticos completos foram realizados para cada zeólita, sendo que foi observada uma perda inferior a $1 \%$ da quantidade em massa do catalisador em cada ciclo.

\section{Espectroscopia Raman}

Espectros de FT-Raman foram adquiridos em um módulo Bruker FRA 106/S acoplado a um espectrômetro Bruker Equinox 55, usando uma cubeta de quartzo de $1 \mathrm{~cm}$ com uma superfície de espelho na direção do espalhamento (128 varreduras e resolução de $4 \mathrm{~cm}^{-1}$ ). A excitação (Nd:YAG) e potência do laser foram de $1064 \mathrm{~nm}$ e 250 $\mathrm{mW}$, respectivamente, e o sinal foi detectado por um detector de Ge resfriado por $\mathrm{N}_{2}$ líquido.

\section{Cromatografia gasosa}

A composição dos ésteres das amostras de biodiesel foi determinada por CG-FID (GC-17A, Shimadzu) utilizando hexano como solvente e uma coluna de poli-dimetil siloxano CBPI PONA-M50-042 da Shimadzu (100 m x 0,25 mm x 0,5 $\mu \mathrm{m})$.

\section{Medidas de HPLC}

O rendimento das reações foi monitorado por cromatografia líquida de alta eficiência (HPLC) em um cromatógrafo da Shimadzu, modelo CTO-20 a , com detector UV-VIS no comprimento de onda de $205 \mathrm{~nm}$, equipado com coluna Shim-PacK VP-ODS (C-18, 250 $\mathrm{mm}, 4,6 \mathrm{~mm}$ de d.i.). A temperatura da coluna foi mantida em $40{ }^{\circ} \mathrm{C}$ e as amostras foram dissolvidas em 2-propanol/hexano (5:4, v:v). A análise quantitativa dos espectros foi feita utilizando-se a soma das áreas dos picos normalizados de ésteres de etila (EEs), entre 4,9 e 8,7 min, e triglicerídeos (TGs), acima de 8,8 min..$^{40}$ 


\section{RESULTADOS E DISCUSSÃO}

A análise estrutural dos catalisadores por DRX mostrou que não houve perda de cristanilidade de ambos os materiais após os procedimentos de impregnação e calcinação (Figura 1). Além disso, não foram observados os picos característicos dos óxidos de bário e estrôncio, indicando que as espécies de $\mathrm{Ba}$ e Sr estão dispersas na superfície da zeólita. No entanto, observou-se uma inversão na intensidade dos picos em $\sim 10^{\circ}$ (plano [220]) e $\sim 12^{\circ}$ (plano [331]) e entre os picos em $\sim 19^{\circ}$ (plano [511] e [333]) e $\sim 20^{\circ}$ (plano [440]) para a zeólita Sr/USY quando comparada à zeólita Ba/USY. Esta inversão pode estar relacionada com uma distribuição não homogênea das espécies de $\mathrm{Sr}$, indicando que os cátions de compensação da rede não estão randomicamente distribuídos e ocupam sítios preferenciais (sítios I' e II) nas supercavidades $\alpha$ da zeólita. ${ }^{41,42}$

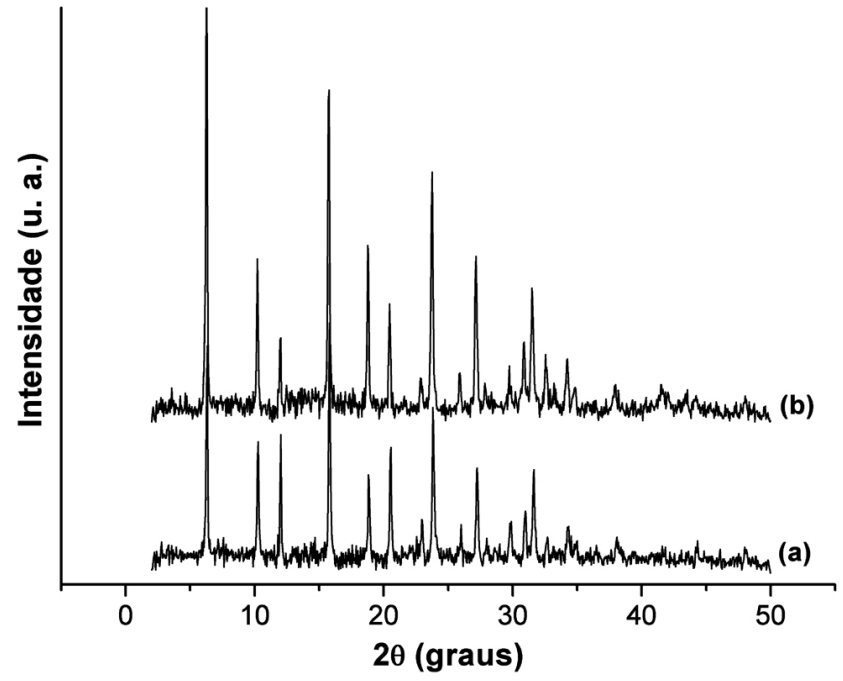

Figura 1. DRX das amostras Ba/USY (a) e Sr/USY (b)

A espectroscopia na região do infravermelho foi utilizada para calcular a razão $\mathrm{Si} / \mathrm{Al}$ intrarreticular das amostras, visto que a banda relativa ao modo de vibração dos anéis duplos (entre 570 e $600 \mathrm{~cm}^{-1}$ ) é considerada a mais sensível à estrutura da zeólita $\mathrm{Y}^{39}{ }^{39}$ Os resultados mostraram que as amostras impregnadas com $\mathrm{Ba}$ e $\mathrm{Sr}$ apresentaram uma maior estabilidade quanto à desaluminização quando comparadas com a zeólita HUSY (Figura 2). A zeólita $\mathrm{NH}_{4}$ USY de partida tem uma razão $\mathrm{Si} / \mathrm{Al}$ da rede igual a 3,7. Após calcinação, formação da zeólita HUSY, apresentou razão $(\mathrm{Si} / \mathrm{Al})_{\text {rede }}=5,2$, enquanto que as zeólitas impregnadas com $\mathrm{Ba}$ e $\mathrm{Sr}$ apresentaram valores de 3,6 e 3,2 , respectivamente. A literatura tem mostrado que zeólitas trocadas com metais alcalinos ou alcalinos terrosos são mais estáveis que as zeólitas na forma amoniacal. ${ }^{43}$

Os catalisadores preparados foram caracterizados quanto à acidez e basicidade por adsorção gasosa de piridina e $\mathrm{CO}_{2}$, respectivamente, seguido de análise térmica. As curvas TG/DTG são ferramentas bastante úteis para caracterizar perfis de DTP de catalisadores. ${ }^{44} \mathrm{Na}$ determinação do número total de sítios das amostras após a adsorção, considerou-se como moléculas sonda quimicamente adsorvidas somente as moléculas dessorvidas acima de $300{ }^{\circ} \mathrm{C} .{ }^{45} \mathrm{Uma}$ comparação dos sítios ácidos obtidos para a zeólita HUSY com os reportados na literatura foi descrita em trabalho anterior, ${ }^{37}$ onde se evidenciou que a razão calculada de piridina adsorvida por $\mathrm{Al}$ estrutural para zeólitas faujasitas pode ser menor que a densidade de átomos de $\mathrm{Al}$ da rede.

Para as zeólitas modificadas, os resultados mostraram que a zeólita Ba/USY apresentou uma menor redução dos sítios ácidos da zeólita HUSY usada como material de partida do que a zeólita $\mathrm{Sr} /$

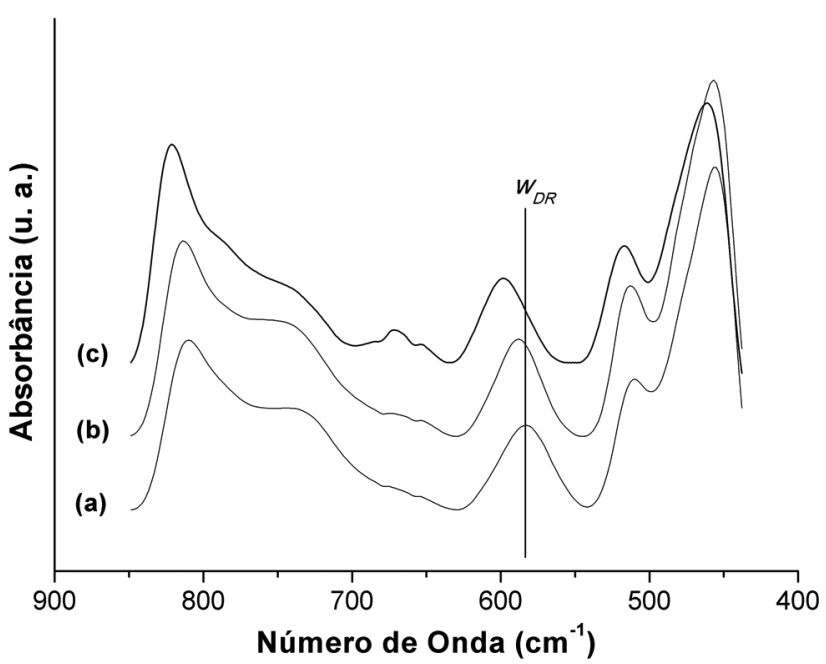

Figura 2. FTIR das amostras $\operatorname{Sr} / U S Y(a), B a / U S Y(b)$ e $H U S Y(c)$

USY (Tabela 1). Embora isso possa significar uma melhor distribuição das espécies de Sr na zeólita HUSY, o número de sítios básicos encontrados para a zeólita Ba/USY foi três vezes maior que o valor encontrado para a Sr/USY. Esse resultado reflete a diferença de basicidade entre estes dois elementos, i.e., $\mathrm{BaO}>\mathrm{SrO}$.

Tabela 1. Número de sítios ácidos e básicos determinados por adsorção de piridina e de $\mathrm{CO}_{2}$ seguida de análises térmicas

\begin{tabular}{ccc}
\hline Amostra & $\mathrm{n}_{\mathrm{Py}}(\mathrm{mmol} / \mathrm{g})^{\mathrm{a}}$ & $\mathrm{n}_{\mathrm{CO}_{2}}(\mathrm{mmol} / \mathrm{g})^{\mathrm{a}}$ \\
\hline $\mathrm{HUSY}$ & $0,88^{\mathrm{b}}$ & - \\
$\mathrm{Ba} / \mathrm{USY}$ & 0,67 & 0,12 \\
$\mathrm{Sr} / \mathrm{USY}$ & 0,57 & 0,04
\end{tabular}

a - Número de sítios determinado por análise térmica entre 300 a $1000{ }^{\circ} \mathrm{C}$. b - Obtido da ref. 37.

Para avaliar a influência da acidez e basicidade obtidas acima, ambas as zeólitas tiveram sua atividade catalítica testada na transesterificação do óleo de soja com etanol para obtenção de ésteres de etila (biodiesel). A determinação da conversão foi realizada por HPLC, utilizando um método reportado por Holčapek e colaboradores,${ }^{40}$ onde a análise dos cromatogramas permitiu uma rápida determinação do rendimento. As Figuras 3 e 4 exibem os cromatogramas de HPLC obtidos para os três ciclos reacionais de transesterificação com as zeólitas Ba/USY e Sr/USY, respectivamente.

Ao analisar os cromatogramas de HPLC para ambas as zeólitas, observa-se que a Sr/USY apresenta uma quantidade superior de triglicerídeos (picos acima de $8,8 \mathrm{~min}) .{ }^{40}$ Além disso, pode-se observar que o biodiesel obtido com a Ba/USY apresenta uma distribuição diferente da obtida para a Sr/USY. Observe que em 7,5 min o biodiesel obtido com a Ba/USY apresenta um pico cuja intensidade aumenta à medida que os ciclos aumentam e, ao mesmo tempo, o pico em $\sim 6,5$ min reduz de intensidade. Essas variações de intensidade não foram observadas para o biodiesel obtido com a zeólita Sr/USY. A análise do produto por espectroscopia Raman mostrou que o biodiesel obtido pela $\mathrm{Ba} / \mathrm{USY}$ apresenta uma redução das bandas relacionadas a duplas ligações $\mathrm{C}=\mathrm{C}$ (Figura 5). Esta observação pode estar diretamente relacionada com o pico observado no cromatograma de HPLC. De fato, a análise por CG-FID mostrou pequenas diferenças na composição de ácidos graxos do biodiesel produzido pela zeólita Ba/USY quando comparado com um biodiesel padrão. Observou-se uma redução da quantidade de ácido oleico de $27,9 \%$ (biodiesel padrão) para $24,5 \%$ (Ba/USY). Sabe-se que o oxigênio molecular reage em condições 


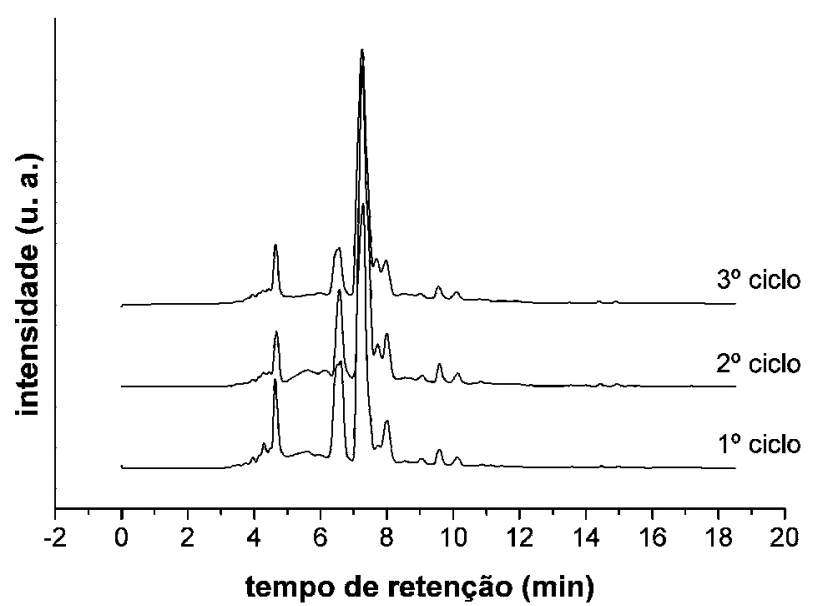

Figura 3. Cromatograma de HPLC para os três ciclos reacionais de transesterificação realizados com a zeólita Ba/USY

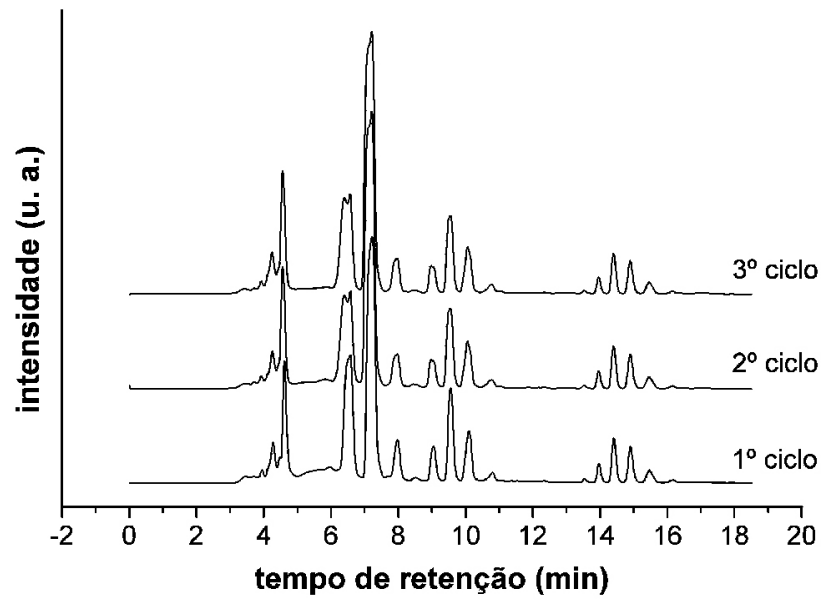

Figura 4. Cromatograma de HPLC para os três ciclos reacionais de transesterificação realizados com a zeólita $\mathrm{Sr} / U S Y$

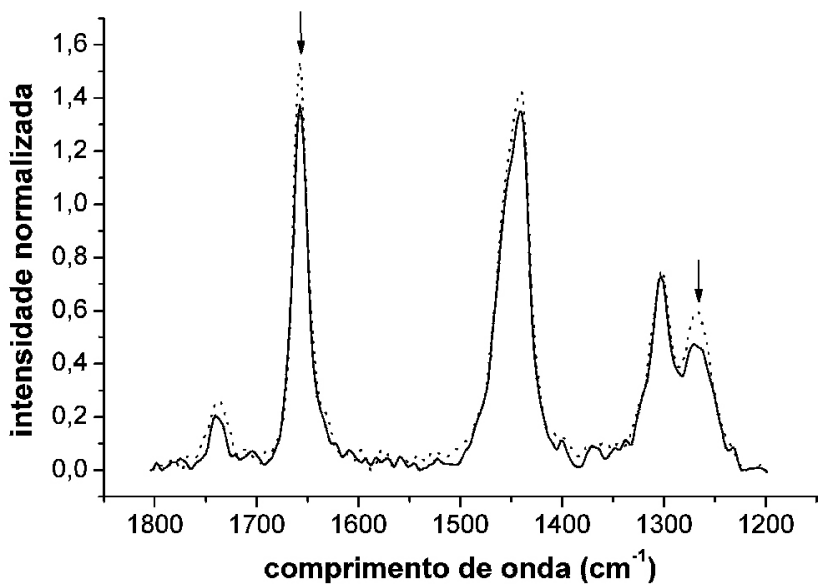

Figura 5. Espectro FT-Raman do biodiesel obtido utilizando as zeólitas Ba/ USY (-) e Sr/USY (....). As setas indicam a redução de bandas relacionadas à dupla ligação $C=C$

brandas com duplas ligações de óleos vegetais. ${ }^{46}$ Desse modo, nas condições reacionais utilizadas neste trabalho $\left(200{ }^{\circ} \mathrm{C}\right.$ e pressão autógena de $\sim 20$ bar) essa reação deve ser favorecida.

A análise quantitativa dos cromatogramas de HPLC foi feita utilizando-se a soma das áreas dos picos normalizados de ésteres de etila (EEs) e triglicerídeos (TGs). ${ }^{40}$ É possível observar através da Tabela 2 os resultados de conversão das zeólitas Ba/USY e Sr/USY nos três ciclos catalíticos realizados e da zeólita HUSY para comparação.

Tabela 2. Conversão de biodiesel das zeólitas HUSY, Ba/USY e Sr/USY nos três ciclos catalíticos realizados para a transesterificação de óleo de soja com etanol

\begin{tabular}{cccc}
\hline Ciclo & $\begin{array}{c}\text { \% biodiesel } \\
\text { HUSY }\end{array}$ & $\begin{array}{c}\text { \% biodiesel }- \\
\text { Ba/USY }\end{array}$ & $\begin{array}{c}\text { \% biodiesel - } \\
\text { Sr/USY }\end{array}$ \\
\hline $1^{\circ}$ & 100 & 97 & 69 \\
$2^{\circ}$ & 98 & 97 & 71 \\
$3^{\circ}$ & 96 & 98 & 71 \\
\hline
\end{tabular}

A análise do primeiro ciclo de transesterificação mostra claramente a atividade superior da zeólita Ba/USY em relação à Sr/USY. Essa diferença pode ser atribuída a dois efeitos distintos: a menor interação entre as espécies de bário formadas e os sítios de Brønsted da zeólita HUSY, possibilitando que uma quantidade maior de sítios ácidos permaneça ativa para a reação e, a geração de uma quantidade de sítios básicos na zeólita $\mathrm{Ba} / \mathrm{USY}\left(0,12 \mathrm{mmol} \mathrm{g}^{-1}\right)$ muito superior à observada para a zeólita Sr/USY $\left(0,04 \mathrm{mmol} \mathrm{g}^{-1}\right)$. Esses dois efeitos combinados parecem tornar a zeólita Ba/USY um catalisador mais eficiente na reação de transesterificação de óleos vegetais que a Sr/ USY. De fato, a análise dos ciclos seguintes mostra que a zeólita $\mathrm{Ba} / \mathrm{USY}$ mantém a mesma alta atividade catalítica observada no primeiro ciclo. Embora a zeólita Sr/USY apresente uma atividade menor que a $\mathrm{Ba} / \mathrm{USY}$, esta também mantém sua atividade catalítica inicial, mostrando que, aparentemente, ambas as amostras não tiveram sua estrutura e sítios comprometidos após reativação em mufla. No entanto, quando comparadas com a HUSY, os resultados mostram um efeito negativo na adição de $\mathrm{Sr}$, enquanto que a Ba/USY mantém uma atividade similar à HUSY, mas sem reduzir a conversão com a sequência de ciclos. Este resultado indica que a distribuição de produtos obtida para os catalisadores está relacionada com altos valores de conversão, visto que a zeólita HUSY apresentou uma distribuição similar à encontrada para a zeólita Ba/USY. Além disso, a análise da acidez dos catalisadores após o terceiro ciclo mostrou que para as zeólitas Ba/USY ( $\left.\mathrm{n}_{\mathrm{Py}}=0,63 \mathrm{mmol} / \mathrm{g}\right)$ e $\mathrm{Sr} / \mathrm{USY}\left(\mathrm{n}_{\mathrm{Py}}=0,57 \mathrm{mmol} / \mathrm{g}\right)$ não houve perda considerável de sítios ácidos, enquanto que para a zeólita HUSY houve uma redução para $0,48 \mathrm{mmol} / \mathrm{g}$. Deste modo, a estabilização da estrutura da zeólita e, consequentemente, dos sítios ativos pela introdução de espécies de bário seria um fator chave para a atividade catalítica observada para a zeólita Ba/USY. Esse estudo mostra que a zeólita USY modificada com Ba é suficientemente ativa na reação de transesterificação.

\section{CONCLUSÕES}

A preparação de zeólitas Ba/USY e Sr/USY através da modificação da zeólita USY comercial levou à geração de sítios básicos. Dentre elas, a zeólita modificada com bário apresentou uma quantidade de sítios básicos três vezes maior que a encontrada para a Sr/USY e uma menor interação com os sítios ácidos de Brønsted da zeólita HUSY. Essas diferenças de número de sítios fizeram com que a zeólita $\mathrm{Ba} /$ USY apresentasse uma conversão superior a 97\% na reação de transesterificação de óleo de soja com etanol para produção de biodiesel. A reutilização de ambos os materiais em ciclos reacionais não provocou redução de atividade catalítica. No entanto, uma distribuição diferente de ésteres de etila para o biodiesel obtido com a zeólita $\mathrm{Ba} / \mathrm{USY}$ foi identificada e assinalada como decorrente da oxidação de duplas ligações $\mathrm{C}=\mathrm{C}$. Esse estudo mostra que a zeólita Ba/USY é suficientemente ativa na reação de transesterificação. 


\section{AGRADECIMENTOS}

Ao Sr. M. B. Alves (LAPREN-UnB) pelas análises de HPLC, ao CNPq, CAPES/PQI, FINATEC, Finep-CTPetro, Finep-CTInfra, UnB-IQ, UnB-IG e FAPDF/SCDT/CNPq.

\section{REFERÊNCIAS}

1. Ma, F.; Hanna, M. A.; Bioresour. Technol. 1999, 70, 1.

2. http://www.biodiesel.org, acessada em Novembro 2010.

3. Knothe, G.; J. Am. Oil Chem. Soc. 1999, 76, 795.

4. The Biodiesel Handbook, Knothe, G.; Gerpen, J. V.; Krahl, J., eds.; American Oil Chemists' Society Press: Champaign, 2005.

5. Meher, L. C.; Sagar, D. V.; Naik, S. N.; Renew. Sustain. Energy Rev. 2006, 10, 248.

6. Schwab, A. W.; Bagby, M. O.; Freedman, B.; Fuel 1987, 66, 1372.

7. Dasari, M. A.; Goff, M. J.; Suppes, G.; J. Am. Oil Chem. Soc. 2003, 80, 189.

8. Schwab, A. W.; Dykstra, G. J.; Selke, E.; Sorenson, S. C.; Pryde, E. H.; J. Am. Oil Chem. Soc. 1988, 65, 1781.

9. Bagby, M. O.; Freedman, B.; Schwab, A. W.; Seed Oils for Diesel Fuels: Sources and Properties, ASAE Paper No. 87-1583; American Society of Agricultural Engineers: St. Joseph, Dec 1987.

10. Schuchardt, U.; Sercheli, R.; Vargas, R. M.; J. Braz. Chem. Soc. 1998, 9, 199.

11. Jackson, M. A.; King, J. W.; J. Am. Oil Chem. Soc. 1996, 73, 353.

12. Freedman, B.; Pryde, E. H.; Mounts, T. L.; J. Am. Oil Chem. Soc. 1984, $61,1638$.

13. Zagonel, G. F.; Peralta-Zamora, P.; Ramos, L. P.; Talanta 2004, 63, 1021.

14. Neto, P. R. C.; Caro, M. S. B.; Mazzuco, L. M.; Nascimento, M. G.; J. Am. Oil Chem. Soc. 2004, 81, 1111.

15. Demirba , A.; Energy Convers. Manage. 2002, 43, 2349.

16. Fukuda, H.; Kondo, A.; Noda, H.; J. Biosci. Bioeng. 2001, 92, 405.

17. Pryde, E. H.; J. Am. Oil Chem. Soc. 1984, 61, 1609.

18. Barnwal, B. K.; Sharma, M. P.; Renew. Sustain. Energy Rev. 2005, 9 , 363.

19. Saka, S.; Kusdiana, D.; Fuel 2001, 80, 225.

20. Shibasaki-Kitakawa, N.; Honda, H.; Kuribayashi, H.; Toda, T.; Fukumura, T.; Yonemoto, T.; Bioresour. Technol. 2006, 98, 416.

21. Suppes, G. J.; Dasari, M. A.; Doskocil, E. J.; Mankidy, P. J.; Goff, M. J.; Appl. Catal., A 2004, 257, 213.
22. Stern, R.; Hillion, G.; Rouxel, J. J.; US patent 6,147,196 2000

23. Furuta, S.; Matsuhashi, H.; Arata, K.; Catal. Commun. 2004, 5, 721.

24. Pan, W. B.; Chang, F. R.; Wei, L. M.; Wu, M. J.; Wu, Y. C.; Tetrahedron Lett. 2003, 44, 331

25. Kim, H. J.; Kang, B. S.; Kim, M. J.; Park, Y. M.; Kim, D. K.; Lee, J. S.; Lee, K. Y.; Catal. Today 2004, 315, 93.

26. Jitputti, J.; Kitiyanan, B.; Rangsunvigit, P.; Bunyakiat, K.; Attanatho, L.; Jenvanitpanjakul, P.; Chem. Eng. J. 2006, 116, 61.

27. Corma, A.; Iborra, S.; Miguel, S.; Primo, J.; J. Catal. 1998, 173, 315.

28. Olson, D. H.; Haag, W. O.; Lago, R. M.; J. Catal. 1980, 61, 390.

29. Giannetto, G.; Zeolitas: Características, Propiedades y Aplicaciones Industriales, $1^{\text {a }}$ ed., Editorial Innovación Tecnológica: Caracas, 1990.

30. Corma, A.; Chem. Rev. 1995, 95, 559.

31. Weisz, P. B.; Frilette, V. J.; J. Phys. Chem. 1960, 64, 382.

32. Csicsery, S. M.; Pure Appl. Chem. 1986, 58, 841.

33. Smart, L.; Moore, E.; Solid State Chemistry, An Introduction, $1^{\text {st }}$ ed., Chapman \& Hall: London, 1992.

34. Luna, F. J.; Schuchardt, U.; Quim. Nova 2001, 24, 885.

35. Barthomeuf, D.; Microporous Mesoporous Mater. 2003, 66, 1.

36. Doskocil, E. J.; Mankidy, P. J.; Appl. Catal., A 2003, 252, 119

37. Ghesti, G. F.; Macedo, J. L.; Parente, V. C. I.; Dias, J. A.; Dias, S. C. L.; Microporous Mesoporous Mater. 2007, 100, 27.

38. Klug, H. P.; Alexander, L. E.; X-Ray Diffraction Procedures, $1^{\text {st }}$ ed., John Wiley \& Sons, Inc.: New York, 1962.

39. Lutz, W.; Rüscher, C. H.; Heidemann, D.; Microporous Mesoporous Mater. 2002, 55, 193

40. Holčapek, M.; Jandera, P.; Fischer, J.; Prokeš, B.; J. Chromatogr., A 1999, 858,13

41. Quayle, W. H.; Lunsford, J. H.; Inorg. Chem. 1982, 21, 97.

42. Ntais, S.; Moschovi, A. M.; Paloukis, F.; Neophytides, S.; Burganos, V. N.; Dracopoulos, V.; Nikolakis, V.; J. Power Sources 2011, 196, 2202.

43. Fujita, S.; Kanai, T.; Oumi, Y.; Sano, T. Em Molecular Sieves: From Basic Research to Industrial Applications; Čejka, J.; Žilková, N.; Nachtigall, P., eds.; Elsevier: Amsterdam, 2005, p. 191.

44. Parrillo, D. J.; Adamo, A. T.; Kokotailo, G. T.; Gorte, R. J.; Appl. Catal. 1990, 67, 107

45. Auroux, A.; Datka, J.; Appl. Catal., A 1997, 165, 473.

46. Muik, B.; Lendl, B.; Molina-Díaz, A.; Ayora-Cañada, M. J.; Chem. Phys. Lipids 2005, 134, 173. 